\title{
Results on radio attenuation length recorded in a Romanian salt mine
}

\author{
A.M. Badescu ${ }^{* a \dagger}$ A. Saftoiu ${ }^{b}$, I. Brancus ${ }^{b}$, D. Stanca ${ }^{b}$, B. Mitrica ${ }^{b}$ \\ ${ }^{a}$ Telecommunication Dept., University POLITEHNICA of Bucharest \\ Bd. Iuliu Maniu, nr. 1-3, Bucharest \\ ${ }^{b}$ Horia Hulubei National Institute for Physics and Nuclear Engineering \\ Strada Reactorului 30, Magurele \\ E-mail: alinabadescueradio.pub.ro
}

\begin{abstract}
We measured the transmission of radio signals through salt in the 'Unirea' salt mine (Slanic Prahova, Romania) at a depth of $208 \mathrm{~m}$, in 3 frequency bands: around $200 \mathrm{MHz}, 400 \mathrm{MHz}$ and $800 \mathrm{MHz}$. We concluded that the higher ones are not suitable for radio detection of cosmic neutrinos. Estimates about the comparative radiowave attenuation after $10 \mathrm{~m}$ of propagation, to $100 \mathrm{~m}$ were made, at $200 \mathrm{MHz}$.
\end{abstract}

35th International Cosmic Ray Conference - ICRC2017

10-20 July, 2017

Bexco, Busan, Korea

${ }^{*}$ Speaker.

$\dagger$ 


\section{Introduction}

High energy neutrinos are secondary particles that are products of interactions of super-GreisenZatsepin-Kuzmin cosmic-rays. Detecting high energy neutrinos would provide unique information about the production and propagation of the particles near and above the GZK cut-off [1].

One indirect detection method for cosmic neutrinos was proposed by Askaryan [2]. He suggested that if a neutrino interacts within a volume of dielectric it will produce a broadband electromagnetic field (including radio frequencies) that can be measured. In order to compensate for the small interaction probability a huge volume of detecting material is required that is found in naturally occurring bulk of dielectrics, such as the salt domes.

In the specific case of a $\mathrm{km}^{3}$ neutrino detector in a salt mine neutrinos are observed indirectly by measuring the radio emission generated by their interaction with the salt. As waves propagate in a non ideal medium before being measured by radio antennas it is impetuous to have first a good geophysical material description for radio wave propagation.

The attenuation of radio waves in salt mines is the first factor that determines: the geometry of a radio detector, the energy threshold of the detector, its resolution and the uncertainties in determining the cosmic neutrino characteristics [3], thus accurate experimental measurements should be performed, and this is the purpose of this work.

We measured the transmission of radio signals through salt in the 'Unirea' salt mine (Slanic Prahova, Romania) at a depth of $208 \mathrm{~m}$. The instrumentation and methods used are described in section 2. Section 3 presents preliminary results and discusses the implications of specific radio frequency instrumentation and phenomena. The last section summarizes the work and discuss further problems to be addressed.

\section{Method and instrumentation}

To measure the attenuation of radio waves in natural salt, 6 cylindrical holes (H1-H6) were drilled in a wall of the chamber. The thickness of the wall is more than $100 \mathrm{~m}$, and there are more than $100 \mathrm{~m}$ of salt below and above the holes. The holes have been drilled parallel to each other, at the same height from the chamber floor, each hole having a total depth of $1 \mathrm{~m}$, and a diameter of $75 \mathrm{~mm}$. The distances between the holes H2-H6 and the reference hole (H1) were: $10 \mathrm{~m}, 50 \mathrm{~m}, 80 \mathrm{~m}, 90 \mathrm{~m}$ and $100 \mathrm{~m}$ (figure 1). The distances have been accurately measured using laser tape measure. The measurements were performed using a Vector Network Analyser

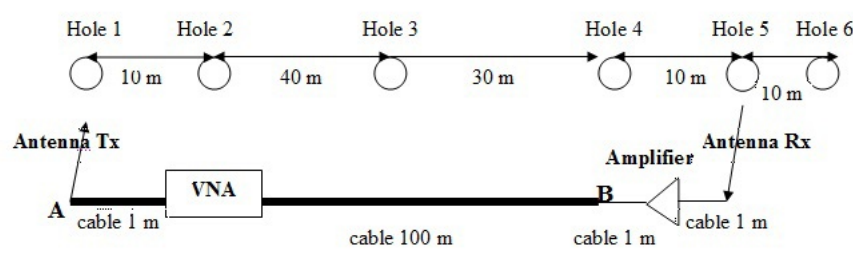

Figure 1: The measurement principle

(VNA), produced by Deviser, model NA7300A, that recorded the $S$-scattering parameters between 
two ports. At each port were connected an emitter antenna (Tx) and a receiver one (Rx). The pairs of antennas used for transmitting and receiving the signals were produced by Kathrein, models K552628 (164-174 MHz - on short $\left.f_{200}\right)$, K737003 (360-430 MHz - $\left.f_{400}\right)$, K751161 (790960 MHz $f_{800}$ ), all omnidirectional dipoles. Antennas' heights were: $993 \mathrm{~mm}, 552 \mathrm{~mm}$, and $348 \mathrm{~mm}$. All ntennas had vertical polarization, input impedance of $50 \Omega$, and gains of $2 \mathrm{dBi}$.

The cables used for connections to the input/output ports of the VNA were a high quality $\mathrm{N}$ type $50 \mathrm{Ohm}$ impedance coaxial cable (EC 400 plus) of $1 \mathrm{~m}$ length (transmitting port) and $100 \mathrm{~m}$ (receiving port). Other 2 coaxial cables (RG 58) of $1 \mathrm{~m}$ length each were used to interconnect the SMA input/output of the amplifier. The amplifier used was produced by Aaronia, model UBBV1, with a gain of about $40 \mathrm{~dB}$ in the $50 \mathrm{MHz}-1 \mathrm{GHz}$ band. The value of the gain at each frequency was extracted from the datasheet provided by the manufacturer.

We calibrated the VNA at the connections between the transmitter antenna feed point (point A on figure 1) and the end of the $100 \mathrm{~m}$ cable (point B on figure 1) using a $50 \Omega$ Open, Short, Match, THRU series of a high precision standard calibration kit.

The frequency-dependent complex ratios $S_{11}(f)$ and $S_{21}(f)$ between the returned signal/ transmitted signal and the emitted signal was measured sequentially at 1601 evenly stepped operating frequencies in each nominal frequency band. The sweep time was set to 2 seconds to increase the accuracy of the measurements, and an averaging factor of 10 was chosen to improve the signal-tonoise ratio. The system had a transmission power of $10 \mathrm{dBm}$ (i.e. $10 \mathrm{~mW}$ ).

$\mathrm{S}$-parameters are basically a means for characterizing $n$-port networks. In our measurement setup, port 1 is the transmitting port, and port 2 - the receiving port. If one considers the impedance normalized travelling voltage waves incident and reflected at port $n$, i.e. $a_{n}=\frac{V_{1}^{+}}{\sqrt{Z_{0 n}}}$ and $b_{n}=\frac{V_{1}^{-}}{\sqrt{Z_{0 n}}}$, with $Z_{0 n}$ being the characteristic impedance associated with port $n$ (here equal to $50 \Omega$ ), the scattering parameters $S_{11}$ and $S_{21}$ from port 1 become [4]:

$$
S_{11}=\left.\frac{b_{1}}{a_{1}}\right|_{a_{2}=0} ; S_{21}=\left.\frac{b_{2}}{a_{1}}\right|_{a_{2}=0}
$$

The quantities $S_{11}$ and $S_{21}$ can be measured using the VNA. $S_{21}$ is associated with voltages that in turn have a direct relationship to far field quantities of antennas.

For all measurement the emitting antenna remained fixed, inserted in hole $\mathrm{H} 1$, and the receiving antenna was moved from one borehole to the other (H2-H6). Thus the instrumental set-up remained the same, and the reflection losses caused by connection of most cables (together with changes in the measurement reference planes) were removed by a null calibration of the VNA.

\section{Results}

Calculation of the total field radiated by an antenna is an old problem with an extensive literature [5],[6]. The space surrounding an antenna is subdivided into three regions: the reactive near-field region, the radiating near-field region, and the far-field region (Fraunhofer region) [5].

Usually, the Fraunhofer region is the one of most practical interest since approximations to simplify the formulation of the fields can be made. The far-field region extends to infinity and its inner boundary is commonly taken to be the radial distance from the antenna aperture as $R_{F}=2 D^{2} / \lambda$ 
[5], where $D$ is the largest dimension of the radiator (here the height), and $\lambda$ is the wavelength. Considering the real part of the permittivity of the salt medium to be equal to 6 (a value typical for pure salt), the far field region extends from about $2 \mathrm{~m}$ (for the $f_{200}$ antenna), $1.4 \mathrm{~m}$ (for $f_{400}$ antenna) and $0.8 \mathrm{~m}$ (for $f_{800}$ antenna). We can conclude that all measurements are done in the far-field region.

The far fields at boreholes $2-6$, from the transmitting halfwave dipole can be approximated by an adaptation of the results in [7]:

$$
E_{\theta}(\theta, r)=C\left(I_{0}, Z_{0}, \Theta, \Phi, h, b, \varepsilon_{r}\right) \times \exp (-i k r) /(4 \pi r)
$$

where $C$ represents an expression of the properties of the emitting antenna in salt, not fully determined analytically $\left(Z_{0}\right.$ is the void characteristic impedance, $I_{0}$ the current feeding the antenna, $b$ is the diameter of the hole where antenna is placed, $h$ is the height of the antenna, $\varepsilon_{r}$-as a measure of the medium surrounding the antenna, etc.). The spherical coordinate system $(r, \Theta, \Phi)$ is defined such as the $o z$ axis is aligned with the antenna, thus $\Theta$ is the angle with respect to the height of the antenna. Since the transmitting and receiving antennas are all parallel, $\Theta$ is equal to $90 \mathrm{deg}$. (the direction of the maximum gain). The time dependence of $\exp (-i 2 \pi f t)$ is suppressed.

The wavenumber $k$ in defined in a lossy ground medium as [8]:

$$
k=2 \pi f \sqrt{\mu \varepsilon_{0}} \sqrt{\varepsilon_{g}-i \frac{\sigma_{g}}{2 \pi f \varepsilon_{0}}}=2 \pi f \sqrt{\mu \varepsilon_{0}} \sqrt{\varepsilon_{r}}
$$

where $\varepsilon_{0}$ is the free-space permittivity, $\mu$ is the permeability of the ground medium (considered free space), $\varepsilon_{g}$ and $\sigma_{g}$ are the relative permittivity and the conductivity of the ground.

The network analyser measures the transmission scattering coefficient $S_{21}$ between the transmitting and receiving antennas. By taking the ratio of two detected fields at different distances from the transmitting antenna (eq. 3.1), one may obtain the average conductivity and permittivity along the propagation line connecting the receiving antennas. This hypothesis holds because the receiving chain (antennas, cables, amplifier etc.) remains identical. Moreover, by taking ratios of measurements the effects of the amplifier and losses induced by the short cables cancel out.

This approach will be detailed it in the following subsection. It is an idealized case, fast and attractive due to its simplicity. Nevertheless, it excludes two effects: the "coupling" of antennas (subsection 3.2), and multiple reflections between the layers of sediments in the salt deposit. The latter will not be discussed in this work since we are interested in the overall characterization of the medium.

\subsection{Simplified model}

For each possible distance between emitting (fixed) and receiving antenna we measured the phase $\phi_{j}$ and amplitude $A_{j}$ of the transmission coefficient:

$$
S_{21, j}=A_{j} \exp \left(i \phi_{j}\right)
$$

The distance between the emitter and receiver is $r_{j}$ (i.e. $r_{j}=10 \mathrm{~m}$ for borehole 2).

To determine the radio attenuation length, adapted from [9], we ca use the formula:

$$
L=\left(r_{6}-r_{2}\right) / \ln \left(\frac{r_{2} S_{21,2}}{r_{6} S_{21,6}}\right)
$$


Note that the $S$ parameters are related to voltages, which are in direct connection to the far field equations. Using formula 3.4 the attenuation length was found to be $29.7 \mathrm{~m}$.

Another observation is that by measuring the $S$ parameter for two distances ( $r_{2}$ and $r_{6}$ ) we can extract the real part and imaginary part of the permittivity of the medium [10]:

$$
\mathfrak{R}\left\{\varepsilon_{r}\right\}=\frac{\left(\phi_{6}-\phi_{2}\right)^{2}-\left[\ln \left(r_{6} A_{6} / r_{2} A_{2}\right)\right]^{2}}{(2 \pi f)^{2} \varepsilon_{0} \mu_{0}\left(r_{6}-r_{2}\right)^{2}} ; \mathfrak{I}\left\{\varepsilon_{r}\right\}=\frac{\left(\phi_{6}-\phi_{2}\right)\left[\ln \left(r_{6} A_{6} / r_{2} A_{2}\right)\right]}{(2 \pi f)^{2} \mu_{0}\left(r_{6}-r_{2}\right)^{2}} \frac{1}{2 \pi f \varepsilon_{0}}
$$

Any set of two measurements can be combined to estimate the properties of the medium. Figure 2 shows results obtained by combining measurements from $r_{3}$ and $r_{2}$, and from $r_{6}$ and $r_{2}$. At 169.4 $\mathrm{MHz}$, results from $50 \mathrm{~m}$ of propagation indicate a value of 5.7196 for $\mathfrak{R}\left\{\varepsilon_{r}\right\}$, and $\mathfrak{I}\left\{\varepsilon_{r}\right\}=0.067$. Figure 3 presents the measured parameters. We have included the whole bandwidth, disregarding
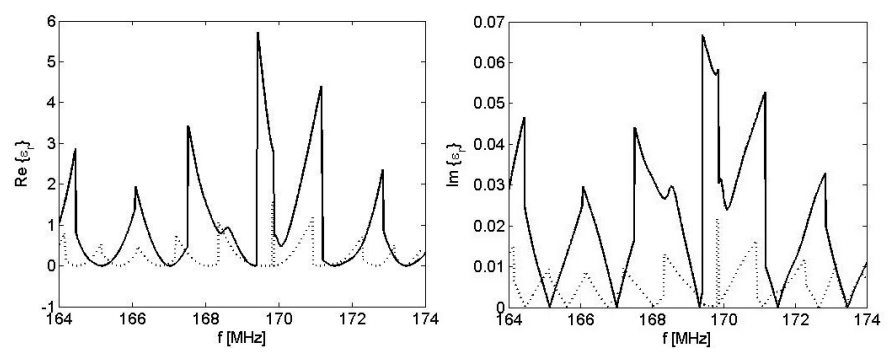

Figure 2: Measurements of the real (left) and imaginary (right) part of the permittivity. The solid line refers to measurements of $50 \mathrm{~m}$ of propagation; interrupted line- $100 \mathrm{~m}$

the $3 \mathrm{~dB}$ characteristic of the antenna. The leaps in the phase occur when material parameters change suddenly causing reflections. These effects are based on electromagnetic wave propagation containing the material parameters. When layering is present, the reflections at the different inter-
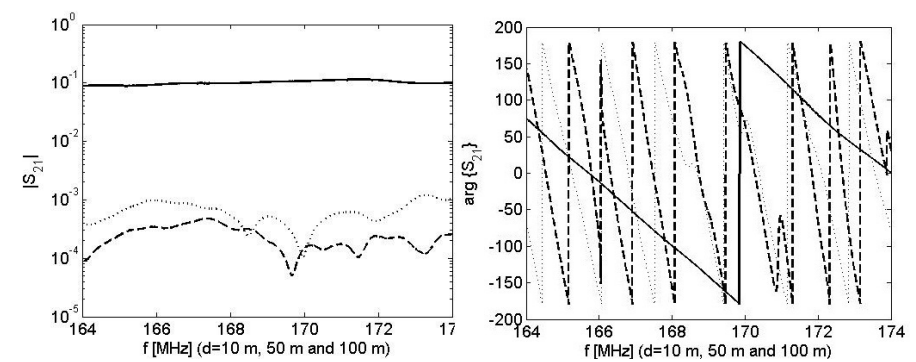

Figure 3: Measurements of the magnitude (left) and argument (right) of the $S_{21}$ transmission parameters. The solid line refers to measurements of $10 \mathrm{~m}$ of propagation; interrupted lines -50 and $100 \mathrm{~m}$

faces may interfere in a constructive or destructive way, leading to a larger or smaller reflection amplitude. The presence of these interferences depends on the ratio between the wavelength $\lambda$ and layer thickness $d$ (usually interferences appear if $d<\lambda / 2$ ), and on the electromagnetic contrast between the layers. If $d=\lambda / 4$, the interferences will me maximum or totally destructive (depending on the sign of the reflection coefficient) [8]. Thus in the case of frequencies in the $f_{200}$ band, any layer of $18 \mathrm{~cm}$ will cause a maximum/minimum interference. Moreover, any layer with a thickness smaller then $36 \mathrm{~cm}$ can produce reflections. 
The magnitude of the reflected wave depends on the difference of constituents (impurities) of each layer. Usually the concentration of such natural impurities is small [11] (thus reflections are small). A special situations is represented by brines in forms of fluid inclusions and primary trapped water. The effect has been estimated in [12]: if the waves travel a distance of $1.1 \mathrm{~cm}$ in brine, about $70 \%$ of their energy will be lost.

For larger frequencies (around $f_{400}$ ), the thickness of layers that produce maximum/ minimum interference is about $8 \mathrm{~cm}$, while for frequencies around $800 \mathrm{MHz}$, it becomes around $3 \mathrm{~cm}$. The walls of the mine chamber clearly show layers that fit all this possible situations. However, due to the formation of the mine deposit, the layers of sediments of few $\mathrm{cm}$ are most frequent, thus the $800 \mathrm{MHz}$ measurement is expected be the most affected. Indeed, measurements have shown that at $800 \mathrm{MHz}$ the waves are attenuated with $\sim 80 \mathrm{~dB}$ after $50 \mathrm{~m}$ of propagation (this result is obtained when an amplifier of $\sim 40 \mathrm{~dB}$ was used at reception). This range of frequencies cannot be used in the context of a cosmic neutrino detector because the large attenuation would require usage of electronic equipment that would exceed the sensibility of the current available technology.

The method described above has been applied to the other measurements at $f_{400}$ and $f_{800}$ and results for the permittivity shown presence of multiple reflections in the medium. The maximum value for $\Re\left\{\varepsilon_{r}\right\}$ is 1.1838 (close to the permittivity for air). It is clear that this simple method does not hold any more and multiple reflections between layers should be incorporated in the model.

\subsection{Coupling of the instrumentation}

The model described in the previous section does not take into account the fact that antennas work differently when boreholed in dielectrics. In order to address it, we have used the signal graph flow [13]. This method perfectly describes the effects of instrumentation using Masons' rules. The equations are too long to be displayed so a few details are given below.

The radio signal emitted by the VNA propagate through a coaxial cable with a fixed characteristic impedance of $50 \Omega$ towards the emission antenna. The antenna is matched when working in air, but not in salt, because the input impedance depends on the radiation resistance, that in turns depends on the medium where the antenna radiates [7]). Thus part of the radio signal will be reflected back toward the VNA.

The part of waves that pass through the antenna are radiated in air (in the hole), and suffer reflections again [14] when they pass in the salt medium, thus re-couple with the antenna, and return to the VNA. The part of the waves that passes in the medium (assumed here continuous) will suffer other reflections when reaching the hole where the reception antenna is positioned, thus return through the medium, a part being reflected when entering the air-medium in the hole, coupling with the antenna etc. Each time the waves pass through the antenna, their are multiplied in the frequency domain by the antenna transfer function $H_{\text {ant }}(f)$ (identical for both emission/reception antennas). The reflections can be described by reflection coefficients $\Gamma$, thus each time when an reflection occurs, the wave's amplitude is multiplied by a reflection (or opposite-transmission) coefficient. The influence of the medium is described by the function $M(r, f)$ (e.g. in air $M=\exp (-i k r) / 4 \pi r$ ).

The $S$ parameters will have the form: $S_{21,11}=S_{21,11}\left(f, H_{\text {ant }}, M, \Gamma_{\text {ant }}, \Gamma_{M}\right)$. We have neglected all the terms of type $M^{2} H_{a n t}^{2}$ in $S_{11}$, and extracted $H_{a n t}=H_{a n t}\left(S_{11}, \varepsilon_{r}, \Gamma_{a n t}\right)$ - here the permittivity appears from the reflection coefficient definition, and $\Gamma_{\text {ant }}$ is the reflection coefficient at the input of the antenna. $H_{a n t}$ can be inserted in the formula for $S_{21}$, which becomes $S_{21}=S_{21}\left(\varepsilon_{r}, \Gamma_{a n t}, M(r)\right)$. 
The effect of the medium can finally be calculated as: $M(r)=M\left(S_{21}, S_{11}, \varepsilon_{r}, \Gamma_{a n t}\right)$. We have considered a non-dispersive medium with $\mathfrak{R}\left\{\varepsilon_{r}\right\}=5.7196$, and $\mathfrak{I}\left\{\varepsilon_{r}\right\}=0.067$. The reflection coefficient at the input of the antenna has not been determined explicitly yet. We considered 1000 random values in the interval $(0,1)$ (for its modulus) and for each determined $M(r)$. In order to decrease the overall effect that $\Gamma_{\text {ant }}$ has, we calculated the ratio $M(10) / M(100)$ obtained from measurements.

Results for the $M(10) / M(100)$ ratio are presented in figure 4 which shows how much the signal is attenuated in $100 \mathrm{~m}$, compared to the attenuation in the first $10 \mathrm{~m}$. Even though not visible, the plot actually shows traces obtained for 1000 values of $\left|\Gamma_{\text {ant }}\right|$. For comparison we have plotted the value of the ratio one would ideally measure in a lossy non-dispersive salt medium (without considering reflection at the input of the antenna and the transfer function of the antenna). We have also determined the antenna transfer function $H$ and concluded that the antenna behaves in salt as a low pass filter, but strongly dependent on $\Gamma_{\text {ant }}$.

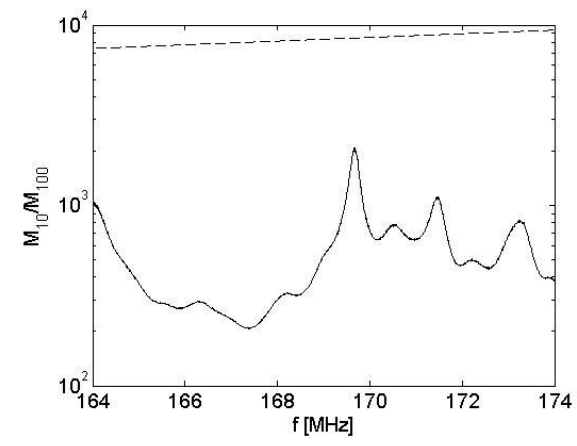

Figure 4: Ratio of attenuation in $100 \mathrm{~m}$ to attenuation in $10 \mathrm{~m}$ extracted from measurements (solid line). The dashed line shows results for propagation in a lossy medium $\left(\Re\left\{\boldsymbol{\varepsilon}_{r}\right\}=5.7196\right.$, and $\left.\mathfrak{I}\left\{\boldsymbol{\varepsilon}_{r}\right\}=0.067\right)$

\section{Conclusions}

The performances of a cosmic neutrino detector in a salt mine are determined by the attenuation length in salt. It determines both the energy threshold of the detector, and the number of detected events. In [9] was stated that a number of 10 GZK events/year requires an attenuation length longer than $100 \mathrm{~m}$. We have measured that for higher frequencies (around $800 \mathrm{MHz}$ ), the medium attenuates the signal with more than $110 \mathrm{~dB}$ in $50 \mathrm{~m}$ of propagation. It is clear that a detector on such high frequencies is not feasible because it would require usage of electronic equipment that would exceed the sensibility of the current available technology.

Comparing the attenuation length (eq. 3.4) with results from [9] in Cote Blanche, at similar frequencies (around $200 \mathrm{MHz}$ ), the attenuation length is about 3 times smaller. However, salt in other deposits in North America showed dielectric constants ranging from 5-7 and loss tangents from 0.015-0.030 or more at $300 \mathrm{MHz}$, implying attenuation lengths below $10 \mathrm{~m}$ [15].

The salt quality in "Unirea" salt mine is clearly poorer compared to Cote Blanche. However we have not taken into account the proximity of the radiation source (antenna) to free space (the mine chamber): the antennas are inserted in the borehole close to the edge of the wall, so part of 
the radiated power will be propagate in the gallery, in air. This should not affect results if ratios of transmissions are considered, because this radiation (equal for all measurements) will bot couple again with the medium. Measurements will be repeated to estimate this effect. Other factors that have not been estimated yet are the complete determination of the antenna transfer function (that should be reduced from measurements), and symmetry of the antenna in the borehole (an offcentre position inside the borehole results in unbalanced antennas; also the emitting and receiving antennas should be parallel for maximum coupling). These effects are small and can be measured.

\section{Acknowledgments}

This project has received funding from the European Research Council (ERC) under the European Union's Horizon 2020 research and innovation programme (grant No. 714637).

\section{References}

[1] J. Alvarez-Muniz, R.A. Vazquez, E. Zas, Calculation Methods for Radio Pulses from High. Energy Showers, Phys. Rev. D 62(2000), 063001

[2] G.A. Askaryan, Coherent radio emission from cosmic showers in air and in dense media, JETP,21(1965), 658

[3] A.M. Badescu, Considerations on an underground neutrino radio detector in salt, JINST, 8 (2013)

[4] S.R. Pennock, P. R. Shepard, Microwave engineering with wireless applications, MacMillan Press LTD, London, 1998

[5] J. Kraus, R. Marhefka, Antennas for all applications, McGraw-Hill, Singapore, 2003

[6] S. Lambot, F. Andre, Full-Wave Modeling of Near-Field Radar Data for Planar Layered Media Reconstruction, TGRS 52 (2014) 5

[7] R. King, G. Smith, Antenna in Matter, ed. The MIT Press, Cambridge, 1981

[8] L. D. Landau, L. P. Pitaevskii, E.M. Lifshitz, Electrodynamics of Continuous Media, Elsevier, 2004

[9] A. Connolly et al., Measurements of radio propagation in rock salt for the detection of high-energy neutrinos, NIMA 599 (2009)

[10] S. Park, H. Choi,J.Ra, Underground Tomogram from Cross Borehole Measurements, Microw Opt Technol Lett. 18(1998)

[11] C. Gomoiu et al., The Vertical Distribution of Rock Salt Thermoluminescence in the Slanic-Prahova (Romania) Halite Deposits, Rom. J. of Phys, 59 (2014)

[12] A.M. Badescu, A. Saftoiu, The effects of naturally occurring impurities in rock salt on radio propagation, Pramana, $\mathbf{8 3}$ (2014)

[13] J.B. Coder, J. M. Ladbury, M. Golkowski, A Two-Port Model for Antennas in a Reverberation Chamber, IEEE TAP 62(2014)

[14] A. S. Negm, I. A. Eshrah, R. I. Badr, Electromagnetic Scattering from a Buried Cylinder using T-Matrix and Signal-Flow-Graph Approach, in proceedings of Ant. and Prop. (EuCAP2015)

[15] P. Saltzberg et al., Measurements of the Suitability of Large Rock Salt Formations for Radio Detection of High Energy Neutrinos, Nucl.Instr.Meth.A 90 (2002) 